\title{
Notification that New Names and New Combinations Have Appeared in Volume 44, No. 2, of the IJSB
}

This listing of names published in a previous issue of IJSB is provided as a service to bacteriology to assist in the recognition of new names and new descriptions. This procedure was proposed by the Judicial Commission [Minute II (ii), Int. J. Syst. Bacteriol. 41:185, 1991]. The names given herein have priority according to the issue of the IJSB in which they were published.

\begin{tabular}{|c|c|c|c|}
\hline Name $^{a}$ & Proposed as: & Authors & $\begin{array}{l}\text { IJSB reference } \\
\text { description: }\end{array}$ \\
\hline Oribaculum & gen. nov. & Moore and Moore & 44(2):189 \\
\hline Oribaculum catoniae & sp. nov. & Moore and Moore & 44(2):189 \\
\hline Catonella & gen. nov. & Moore and Moore & $44(2): 189$ \\
\hline Catonella morbi & sp. nov. & Moore and Moore & 44(2):189 \\
\hline Hallella & gen. nov. & Moore and Moore & 44(2):190 \\
\hline Hallella seregens & sp. nov. & Moore and Moore & $44(2): 190$ \\
\hline Johnsonella & sp. nov. & Moore and Moore & $44(2): 190$ \\
\hline Johnsonella ignava & sp. nov. & Moore and Moore & 44(2):190 \\
\hline Dialister & gen. nov., nom. rev. & Moore and Moore & 44(2):191 \\
\hline Dialister pneumosintes & sp. nov. & Moore and Moore & 44(2):191 \\
\hline Couchioplanes & gen. nov. & Tamura et al. & 44(2):199 \\
\hline Couchioplanes caeruleus subsp. caeruleus & sp. nov., subsp. nov. & Tamura et al. & $44(2): 200$ \\
\hline Couchioplanes caeruleus subsp. azureus & subsp. nov. & Tamura et al. & $44(2): 201$ \\
\hline Porphyromonas canoris & sp. nov. & Love et al. & 44(2):207 \\
\hline Clostridium hydroxybenzoicum & sp. nov. & Zhang et al. & $44(2): 218$ \\
\hline Lactobacillus pontis & sp. nov. & Vogel et al. & $44(2): 228$ \\
\hline Actinocorallia & gen. nov. & Iinuma et al. & $44(2): 233$ \\
\hline Actinocorallia herbida & sp. nov. & Iinuma et al. & $44(2): 233$ \\
\hline Burkholderia plantarii (basonym Pseudomonas plantarii) & comb. nov. & Urakami et al. & $44(2): 242$ \\
\hline Burkholderia glumae (basonym Pseudomonas glumae) & comb. nov. & Urakami et al. & $44(2): 242$ \\
\hline Burkholderia vandii & sp. nov. & Urakami et al. & $44(2): 242$ \\
\hline Bacillus mojavensis & sp. nov. & Roberts et al. & $44(2): 263$ \\
\hline Kutzneria & gen. nov. & Stackebrandt et al. & $44(2): 267$ \\
\hline $\begin{array}{l}\text { Kutzneria viridogrisea (basonym Streptosporangium } \\
\quad \text { viridogriseum) }\end{array}$ & comb. nov. & Stackebrandt et al. & $44(2): 268$ \\
\hline $\begin{array}{l}\text { Kutzneria kofuensis (basonym Streptosporangium } \\
\text { viridogriseum subsp. kofuense) }\end{array}$ & comb. nov. & Stackebrandt et al. & 44(2):268 \\
\hline Kutzneria albida (basonym Streptosporangium albidum) & comb. nov. & Stackebrandt et al. & 44(2):268 \\
\hline Streptosporangium & emend. & Stackebrandt et al. & $44(2): 268$ \\
\hline Turicella & gen. nov. & Funke et al. & $44(2): 272$ \\
\hline Turicella otitidis & sp. nov. & Funke et al. & $44(2): 272$ \\
\hline Planomonospora sphaerica & sp. nov. & Mertz & $44(2): 278$ \\
\hline Planomonospora alba & sp. nov. & Mertz & $44(2): 280$ \\
\hline Pseudoamycolata pro synon Pseudonocardia & synon. & Warwick et al. & 44(2):301 \\
\hline Pseudonocardia & emend. & Warwick et al. & 44(2):301 \\
\hline $\begin{array}{l}\text { Pseudonocardia halophobica (basonym Pseudoamycolata } \\
\text { halophobica) }\end{array}$ & comb. nov. & Warwick et al. & $44(2): 301$ \\
\hline Capnocytophaga haemolytica & sp. nov. & Yamamoto et al. & $44(2): 327$ \\
\hline Capnocytophaga granulosa & sp. nov. & Yamamoto et al. & $44(2): 327$ \\
\hline Luteococcus & gen. nov. & Tamura et al. & $44(2): 355$ \\
\hline Luteococcus japonicus & sp. nov. & Tamura et al. & $44(2): 355$ \\
\hline Methanosarcina & emend. & $\mathrm{Ni}$ et al. & $44(2): 359$ \\
\hline Methanosarcina siciliae (basonym Methanolobus siciliae) & comb. nov. & $\mathrm{Ni}$ et al. & $44(2): 359$ \\
\hline Kingella oralis (not $K$. orale) & corrig. & Dewhirst et al. & $44(2): 376$ \\
\hline
\end{tabular}

